\title{
Decoding the brain through research-the future of brain health
}

\author{
David Z Wang and colleagues look at the latest advances in brain research and how they might \\ affect treatment of brain disorders
}

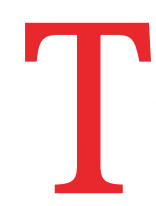

he world has come a long way in solving the mystery of the brain, understanding its fundamental role in human consciousness and discovering methods to treat its disorders. In The Sacred Disease in $\sim 430$ BC, Hippocrates wrote that the brain served to house the ventricles, whose main purpose was to be a container and transit point for the breath or air (pneuma) from outside the body-the force that brought to life our joys, pleasures, laughter, and grief. Thus, the brain was a reservoir for an animated substance that produced the human experience of consciousness and personality rather than the source of that activity itself. ${ }^{1}$ Our knowledge of the brain and its functional complexity remained at the level of three ventricles where our soul lies (Nemesius, da Vinci) for hundreds of years until modern neuroscience began to uncover the fine network of neuronal circuits that made up the solid substance of the brain.

With the advent of modern neuroimaging, the complex structure of the brain has been brilliantly revealed, and this has helped greatly in the treatment of many brain related disorders. Other articles in this series have provided updates on a wide range of topics, including neurodegenerative diseases, mental disorders, cerebrovascular diseases, epilepsy, monogenic neurological

\section{KEY MESSAGES}

- In the past decade, neuroscience and brain research have entered into a new era

- It is now possible to understand brain physiology and pathophysiology better through direct and in vivo observation of live brain

- In the coming years, artificial intelligence will likely be part of brain science and assist or replace certain brain function

- Genetic or protein alterations may provide a cure for many brain disorders in the near future diseases, and in vivo brain function testing. ${ }^{2-6}$ With help from gross anatomy to electronic microscopy, tissue staining to profiling, cell physiology, and synaptic chemistry, neuroscientists have elucidated the mechanisms and pathophysiology of many common brain diseases. For example, trinucleotide repeat expansion is now known to be responsible for many genetically inherited degenerative diseases such as Huntington's disease, and amyloid precursor gene or presenilin gene mutations can cause Alzheimer's disease.

On the other hand, despite centuries of discovery on mechanisms of brain disease, treatment options remain limited. Most treatments still provide only alleviation of symptoms, though recent breakthroughs in gene therapy such as onasemnogene abeparvovec-xioi to treat children with spinal muscular atrophy ${ }^{7}$ and reperfusion therapy for acute ischaemic stroke hold the promise to truly revolutionise treatment for neurological disease. While options are available to modify disease expression with medications-such as in the treatment of Parkinson's disease, multiple sclerosis, and epilepsy-we are far from curing them.

Entering the 21st century, perhaps we now have better ways to understand the mechanism of those brain disorders that are still a mystery and find the precise treatment. The key will likely be interdisciplinary research. Many ongoing brain health research programmes have already been multidimensional, combining neurobiology, physics, engineering, big data science, and artificial intelligence.

\section{Imaging advances}

In the future, it is likely that humans will be able to live longer, and do so with augmented capabilities supported by machinehuman interactions. One exciting advance is new ways of observing in vivo brain-wide activities at the cellular level. A real time, ultra-large scale, high resolution (RUSH) macroscope has recently been developed that can provide video-rate gigapixel imaging of biological dynamics at centimetre scale and micrometre resolution, with a data throughput of up to 5.1 gigapixels a second. ${ }^{8}$ RUSH has enabled in vivo functional imaging of neural networks across the whole mouse brain at single dendrite resolution and brain-wide tracking of leucocytes during pathological processes, and the technology opens up a new horizon for large scale brain imaging to study various brain diseases at a systematic level. ${ }^{8}$

Another example is the better understanding of the precise number of brain cells needed to complete a particular task. By constructing an explicit model of face selective cells that could decode an arbitrary realistic face from face cell responses and predict the firing of cells in response to an arbitrary realistic face, Chao and colleagues identified that macaques require only 200 cells to remember a face. ${ }^{9}$ These findings have far reaching significance. For the first time, a specialised task of the brain can be attributed to a specific number and type of brain cells in a specific circuit. This may allow scientists to build artificial models of explicit brain functions and experiment with mechanisms of injury and repair at a cellular or molecular level. Such mapping may aid our understanding of brain function and recovery and guide the rebuilding of brain circuits or resection of dysfunctional brain cells rather than whole tissues. It may also help us pinpoint the cells and circuits that are responsible for addictive behaviours, from smoking to substance use disorders to gambling.

\section{Resilience and plasticity of brain cells}

The common belief is that when a brain has been removed, brain death is imminent. However, such belief has recently been shattered. Sestan and colleagues collected brains of 6-8 month old pigs four hours after death and bathed them in specialised perfusate solutions. They found that brain cells and synapses of certain areas of brain began to recover and show signs of cellular activities. ${ }^{10}$ Their finding suggests that there may be a late window of treatment after onset of brain anoxia when brain tissue can recover, analogous to the 
benefit of late window thrombectomy. This discovery has taught us that brain cells can survive and recover after loss of circulation, and that favourable conditions may preserve a reservoir of resilient brain cells that are slow progressors to ischaemic necrosis.

Evidence is also emerging on how brain cells can adapt. A recent report of functional neuronal connectivity in adults without apparent loss of function after brain hemispherectomy sheds new light on brain plasticity. The study provides the first comprehensive analysis of whole brain functional connectivity across the full repertoire of resting state networks after hemispherectomy and shows preservation of resting state networks but an increase in internetwork connectivity with other functional brain networks. When hemispheric resection occurred in patients younger than 11 , the retained hemisphere was able to protect the jeopardised functions by enhancing cellular interaction and synaptic activity. ${ }^{11}$

\section{Harnessing the power of big data}

Artificial intelligence (AI) has been widely applied in clinical diagnosis and patient monitoring. Recent studies have attempted to classify or detect Alzheimer's disease and other cognitive impairment, ${ }^{12}{ }^{13}$ acute neurological events, ${ }^{14-18}$ focus of epilepsy, autism spectrum disorder, and attention deficit/hyperactivity disorder by using deep learning based algorithms. The data in these AI models include not only medical images but also clinical scores, in vitro diagnostic test results, and other functional and structure information. ${ }^{19-25}$ These studies showed high sensitivity and specificity from their test set, and work is ongoing on how to incorporate the routine use of these AI systems into a clinical setting.

The lack of a large dataset from multiple centres, the limited coverage of a disease spectrum, and unclear risk of using AI are major limitations of these blackbox systems. In contrast, Wang and colleagues have recently proposed a "vascular aware" unsupervised learning technique, VasNet, ${ }^{26}$ which provides the end users with explainable images, including both vascular structures and multidimensional features such as anatomical, physiological, biochemical, and cellular details. The enriched outputs could augment human decision making on treating vascular diseases and contribute to the emergence of the next generation of healthcare engineering.
The US Food and Drug Administration has already approved several automatic quantitative measurement software systems for disease classification (eg, NeuroQuant, Quantib, RAPID). Brain morphometry analysis software can automatically examine segments of brain tissue and detect minute changes. This technology can help early detection of degenerative brain diseases by comparing the results from individuals with a large dataset and images of healthy people. To take racial differences in the brain into account, some Asian companies have developed software based on datasets acquired from Asian populations (http:// quant-health.com). Use of a deep learning based segmentation algorithm could improve the accuracy and test-retest stability in segmenting and measuring the volume of brain structure, abnormal lesions, perfusion deficit area, and other characteristics. The resulting quantified values could be used to assign a clinical score automatically, avoiding the variation arising from subjective measurement and interobserver inconsistency.

AI algorithms can also objectively analyse the data collected from a depth camera or wearable devices, assess behaviour, and evaluate facial expressions. ${ }^{27-29}$ The quantified values produced would not be affected by the physicians' experiences, and errors can be avoided since the spatialtemporal resolution of the hardware is much smaller than visual evaluation by humans. Such early detection may allow treatment of a disease before a person shows clinical signs of brain dysfunction. Quantified measurements can be used as biomarkers to monitor the progress of the disease and help evaluate the efficacy of precision therapy.

\section{Prospect of cure}

One of the potential ways of curing a brain disorder is to correct its diseased protein structure. Many neurological diseases are caused by misfolded proteins, including Huntington's, Parkinson's, and Alzheimer's disease. AlphaFold, a Google company, has successfully predicted a protein structure by using large genomic data. The 3D models of proteins that AlphaFold generates are far more accurate than any that have come before-making significant progress on one of the core challenges in biology. The ability to predict a protein's shape from its DNA sequence is useful to scientists because it is fundamental to understanding its role within the body, as well as diagnosing and treating diseases believed to be caused by protein misfolding. ${ }^{30}$

We have entered into an exciting new era of brain science research and discovery. With the advent of AI, advanced imaging, genomics, psychosocial analytics, and protein engineering we may be closer than ever to new precision medicine approaches to treat many brain disorders.

Contributors and sources: DZW drafted the first manuscript. LHS, TYQ, and QHD critically reviewed and revised the manuscript. DZW is an expert in stroke clinical research. LHS is an expert in neuroscience research and stroke care quality improvement. TYQ is an expert in big data and artificial intelligence. QHD is an expert in brain research and artificial intelligence.

Competing interests: We have read and understood BMJ policy on declaration of interests and declare that we have no competing interest.

Provenance and peer review: Commissioned; externally peer reviewed.

This article is part of a series launched at the Chinese Stroke Association annual conference on 10 October 2020, Beijing, China. Open access fees were funded by the National Science and Technology Major Project. The BM/ peer reviewed, edited, and made the decision to publish these articles.

David Z Wang, professor

Lee H Schwamm, professor ${ }^{2}$

Tianyi Qian, professor ${ }^{3,4}$

Qionghai Dai, professor ${ }^{4}$

${ }^{1}$ Neurovascular Division, Department of Neurology, Barrow Neurological Institute, St Joseph Hospital and Medical Center, Phoenix, AZ, USA

${ }^{2}$ Comprehensive Stroke Center, Massachusetts General Hospital and Harvard Medical School, Boston, MA, USA

${ }^{3}$ Department of Public Health, School of Medicine, Tsinghua University, Beijing

${ }^{4}$ Tencent Healthcare, Tencent AIMIS, Shenzhen, China ${ }^{5}$ Department of Automation and Institute for Brain and Cognitive Science, Tsinghua University, Beijing, China Correspondence to: David Wang david.wang@dignityhealth.org

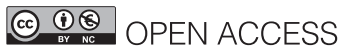

This is an Open Access article distributed in accordance with the Creative Commons Attribution Non Commercial (CC BY-NC 4.0) license, which permits others to distribute, remix, adapt, build upon this work non-commercially, and license their derivative works on different terms, provided the original work is properly cited and the use is non-commercial. See: http://creativecommons.org/ licenses/by-nc/4.0/.

\section{Check for updates}

1 Robert LM. The brain takes shape: an early history. Oxford University Press, 2004.

2 Shi J, Sabbagh MW, Vellas B. Alzheimer's disease beyond amyloid: strategies for future therapeutic interventions. BMJ 2020;371:m3684. doi:10.1136/ bmj.m3684 
3 Chen R, Cui Z, Capitao L, et al. Precision biomarkers for mood disorders based on brain imaging. BMJ 2020;371:m3618. doi:10.1136/bmj.m3618

4 Chen Z, Rollo B, Baker A, et al. New era of personalised epilepsy management. BMJ 2020;371:m3658. doi:10.1136/bmj.m3658

5 Yeusong $\mathrm{O}$, Ku Hm Wardkaw JM, Wang Y. A new dawn of preventing dementia by preventing cerebrovascular diseases. BMJ 2020;371:m3692. doi:10.1136/bmi.m3692

6 Zhang I. Li D, Xiao D, Couldwell WT, Ohata K. Improving brain health by identifying structurefunction relations in patients with neurosurgical disorders. BMJ 2020;371:m3690. doi:10.1136/bmj. m3690

7 FDA approves innovative gene therapy to treat pediatric patients with spinal muscular atrophy, a rare disease and leading genetic cause of infant mortality. Press release, 24 May 2019. https://www.fda.gov/ news-events/press-announcements/fda-approvesinnovative-gene-therapy-treat-pediatric-patientsspinal-muscular-atrophy-rare-disease\#: :text=The\%20 U.S.\%20Food\%20and\%20Drug,genetic\%20 cause $\% 20$ of\%20infant $\% 20$ mortality

8 Fan J, Suo J, Wu J, et al. Video-rate imaging of biological dynamics at centimetre scale and micrometre resolution. Nat Photonics 2019:13:80916. doi:10.1038/s41566-019-0474-7

9 Chang L, Tsao DY. The code for facial identity in the primate brain. Cell 2017;169:1013-1028.e14. doi:10.1016/j.cell.2017.05.011

10 Vrselja Z, Daniele SG, Silbereis J, et al. Restoration of brain circulation and cellular functions hours postmortem. Nature 2019;568:336-43. doi:10.1038/ s41586-019-1099-1

11 Saplakoglu Y. How the brain still works when half of it is missing. Live Science, 22 Nov 2019. https:// www.livescience.com/hemisphere-removed-brainplasticity.html

12 Suk H-I, Shen D. Deep learning-based feature representation for $\mathrm{AD} / \mathrm{MCl}$ classification. Med Image Comput Comput Assist Interv 2013;16:583-90. doi:10.1007/978-3-642-40763-5_72
13 Payan A, Montana G. Predicting Alzheimer's disease: a neuroimaging study with 3D convolutional neural networks. arXiv 2015:1502.02506. [Preprint.] https://arxiv.org/abs/1502.02506.

14 Titano JJ, Badgeley M, Schefflein J, et al. Automated deep-neural-network surveillance of cranial images for acute neurologic events. Nat Med 2018;24:1337-41. doi:10.1038/s41591-018-0147-y

15 Zech J, Pain M, Titano J, et al. Natural language based machine learning models for the annotation of clinical radiology reports. Radiology 2018;287:57080. doi:10.1148/radiol.2018171093

16 Arbabshirani MR, Fornwalt BK, Mongelluzzo GJ, et al. Advanced machine learning in action: identification of intracranial hemorrhage on computed tomography scans of the head with clinical workflow integration. NPJ Digit Med 2018;1:9. doi:10.1038/s41746-0170015-z

17 Chilamkurthy S, Ghosh R, Tanamala S, et al. Deep learning algorithms for detection of critical findings in head CT scans: a retrospective study. Lancet 2018;392:2388-96. doi:10.1016/S01406736(18)31645-3

18 Lee $H$, Yune S, Mansouri M, et al. An explainable deep-learning algorithm for the detection of acute intracranial haemorrhage from small datasets. Nat Biomed Eng 2018;5:6. doi:10.1038/s41551-0180324-9.

19 Gupta A, Ayhan M, Maida A. Natural image bases to represent neuroimaging data. Proceedings of 30 th international conference on machine learning. Vol 28. Atlanta, GA. 2013:987-94.

20 Li F, Tran L, Thung K-H, Ji S, Shen D, Li J. Robust deep learning for improved classification of $A D / M C l$ patients. Machine learning in medical imaging. Springer International, 2014:240-7.

21 Liu S, Liu S, Cai W, Pujol S, Kikinis R, Feng D. Early diagnosis of Alzheimer's disease with deep learning. 2014 IEEE 11th international symposium on biomedical imaging. 29 Apr-2 May, 2014:1015-8. https://ieeexplore.ieee.org/document/6868045.

22 Liu S, Liu S, Cai W, et al, ADNI. Multimodal neuroimaging feature learning for multiclass diagnosis of Alzheimer's disease. IEEE Trans Biomed Eng 2015;62:1132-40. doi:10.1109/ TBME.2014.2372011

23 Suk H-I, Lee S-W, Shen D, Alzheimer's Disease Neuroimaging Initiative. Latent feature representation with stacked auto-encoder for $A D /$ MCl diagnosis. Brain Struct Funct 2015:220:841-59. doi:10.1007/s00429-013-0687-3

24 Sarraf S, Tofighi G. Classification of Alzheimer's disease using fMRI data and deep learning convolutional neural networks. 2016. https://arxiv. org/abs/1603.08631.

25 Suk H-I, Lee S-W, Shen D, Alzheimer's Disease Neuroimaging Initiative. Deep sparse multi-task learning for feature selection in Alzheimer's disease diagnosis. Brain Struct Funct 2016;221:2569-87. doi:10.1007/s00429-015-1059-y

26 Wang Y, Ji M, Jiang S, et al. Augmenting vascular disease diagnosis by vasculatureaware unsupervised learning. Nature Machine Intelligence 2020;2:337-46. doi:10.1038/s42256 020-0188-z

27 Khan MH, Helsper J, Farid MS, Grzegorzek M. A computer vision-based system for monitoring Vojta therapy. Int J Med Inform 2018;113:85-95. doi:10.1016/j.ijmedinf.2018.02.010

28 Rovini E, Maremmani C, Cavallo F. How wearable sensors can support Parkinson's disease diagnosis and treatment: a systematic review. Front Neurosci 2017;11:555. doi:10.3389/ fnins.2017.00555

29 Mark MD, Omar AU, Yan Z, et al. Comparison of human and machine learning based facial weakness detection. Stroke 2019;50(Suppl 1):tp274.

30 Evans R, Jumper J, Kirkpatrick J, et al. De novo structure prediction with deep-learning based scoring. In: Thirteenth critical assessment of techniques for protein structure prediction. Abstracts, 1-4 December 2018.

Cite this as: $B M J$ 2020;371:m3735

http://dx.doi.org/10.1136/bmj.m3735 